\title{
Adaptação do índice de massa corporal humano para cães
}

\author{
Adaptation of human body mass index for dogs
}

\author{
Daniel Curvello de Mendonça Muller ${ }^{I}$ João Eduardo SchosslerII Maicon Pinheiro ${ }^{\text {III }}$
}

\section{RESUMO}

O índice de massa corporal (IMC) é amplamente utilizado por médicos para quantificar a massa corporal de pessoas adultas. $O$ índice elevado de gordura, ou seja, o peso em excesso, está relacionado com problemas cardiovasculares, infertilidade, diabetes, artrite, dificuldade em se locomover ou respirar e prejuízos na cicatrização de feridas. Apesar de todo o estudo que se tem a respeito desses problemas, não há um método preciso e objetivo para se quantificar o excesso de peso em cães. A proposta deste trabalho foi determinar um índice de massa corporal canino (IMCC), a partir do IMC existente para humanos. Foram medidos e pesados 246 animais, sem raça definida e com diferentes condições corporais. Para dar validade ao índice de massa corporal obtido, os animais também foram avaliados por dois veterinários segundo os padrões subjetivos, já existentes. Verificou-se que valores do IMCC entre 11,8 e $15 \mathrm{~kg} \mathrm{~m}^{-2}$ refletem o peso ideal para cães de porte médio, cujo tipo físico possui média de peso entre 10 e $25 \mathrm{~kg}$.

Palavras-chave: índice de massa corporal, cães, obesidade.

\section{ABSTRACT}

The index of corporal mass (ICM) is broadlly used to measure the corporal mass of adults. A high fat index is related to heart and circulation problems, infertility, diabetes, arthritis, difficulty to move around or breath and problems with cicatrization. Even though there are several studies about these problems, there is no specific method to evaluate weight excess in dogs objectively and precisely. The purpose of this research was to determine an index of corporal mass for dogs based on the existing IMC for human beings. Two hundred and forty six dogs without defined-breed and with different body conditions were analysed. They were not only measured and weighed, but also evaluated by two distinct veterinarians based on real subjective patterns, which can determine the corporal score. These procedures enabled the acknowledgement of the attained index. The conclusion was that IMCC between 11,8 and $15 \mathrm{~kg} \mathrm{~m}^{-2}$ represent the ideal pattern for dogs of average size that weigh between 10 and 25kilos.

Key words: index of corporal mass, dogs, obesity.

\section{INTRODUÇÃO}

O índice de massa corporal (IMC) é um indicador muito usado por médicos e pesquisadores para avaliar a "normalidade" do peso corporal de uma pessoa e é obtido a partir da divisão da massa corporal (peso) pelo quadrado da estatura (ANJOS, 1992; MCARDLE et al., 2003). Aimportância deste índice está na sua relação curvilínea com a mortalidade, ou seja, à medida que o IMC aumenta devido ao peso excessivo, o mesmo ocorre com o aumento do risco para complicações cardiovasculares, cânceres, diabetes, cálculos vesiculares e osteoartrite (MONTEIRO et al., 2000; MCARDLE et al., 2003). Além de detectar o excesso de peso, o IMC alerta para os riscos de doenças ocasionadas pela desnutrição.

O acúmulo excessivo de gordura corpórea é a condição que caracteriza a obesidade, acometendo não somente os seres humanos como também os animais de companhia, principalmente os cães. A obesidade provoca em seus portadores variadas disfunções fisiológicas e, diante disto, é evidente o prejuízo à qualidade de vida do animal (BURKHOLDER \& TOLL, 2000). Assim como a obesidade, a desnutrição

\footnotetext{
IPrograma de Pós-graduação em Medicina Veterinária, Universidade Federal de Santa Maria (UFSM). Rua ACDO Rigoberto Duarte, 70, ap 101, 97060-030, N. Sr de Lourdes, Santa Maria, RS, Brasil. E-mail: cmdaniel@terra.com.br. Autor para correspondência. "Departamento de Clinica de Pequenos Animais, UFSM, Santa Maria, RS, Brasil.

${ }^{\text {II } C u r s o ~ d e ~ M e d i c i n a ~ V e t e r i n a ́ r i a, ~ U F S M, ~ S a n t a ~ M a r i a, ~ R S, ~ B r a s i l . ~}$
} 
(atendimento energético inferior às necessidades) pode provocar alterações relevantes na fisiologia do paciente (MONDINI \& MONTEIRO, 1998).

Segundo MENTZEL et al. (2006), um animal obeso tem risco cinco vezes maior de transtornos articulares. Além disso, a obesidade pode ser considerada como fator de risco significativo para as seguintes doenças: diabetes mellitos, doenças pulmonares, doenças hepáticas, hiperlipidose, artrose e complicações na cicatrização de feridas.

O sobrepeso ou a desnutrição em cães geralmente não são difíceis de serem reconhecidos, mas o diagnóstico correto requer a identificação dos níveis de risco e isso necessita de algumas formas de quantificação para maior exatidão do diagnóstico. Sobretudo, busca-se uma maneira de determinar, de fato, quantos quilos o animal necessita perder ou ganhar.

Alguns autores sugerem que o cão está no porte ideal quando as costelas forem facilmente palpáveis e, quando visto de cima, o animal apresentar forma de ampulheta. Por sua vez, abdômen aumentado a partir da última costela, depósitos de gorduras evidentes bilateralmente à inserção da cauda, bacia, região inguinal e gradil costal de difícil palpação são indicativos de excesso de peso (NELSON \& COUTO, 2001).

SAIOLA (2001) sugere a palpação de pregas cutâneas na região das costelas. Ao agarrar uma prega muito grossa, o animal está acima do peso. Segundo este pesquisador, o aumento da massa de gordura ao redor do pescoço do cão também é um parâmetro usado para diagnosticar o acúmulo de adiposidade. Em 1997, criou-se um sistema para avaliação de condição corporal em caninos, também baseado na inspeção e palpação do paciente, porém dividindo os escores corporais de um a nove (Tabela 1), o que diminuiu em partes a subjetividade (LAFLAMME, 1997).

O IMC é um método reconhecido pela Organização Mundial de Saúde, no qual uma pessoa adulta pode obter uma indicação, com bom grau de acuidade, se está abaixo do peso, no peso ideal, acima do peso ou obeso. A fórmula para calculá-lo é dividir o peso pelo quadrado da altura (IMC=peso altura-2 ${ }^{-2}$ e a sua interpretação é feita da seguinte forma: quando o IMC for inferior a 18,5 o indivíduo está abaixo do peso. Os valores entre 18,5 e 25 representam o índice da normalidade; entre 25 e 30 representam o índice acima do peso e os valores acima de 30 representam a obesidade (STELLA, 2002; MONTILLA et al., 2003).

Cabe salientar que este índice é apenas um indicador e não determina de forma inequívoca a obesidade de uma pessoa, pois desconsidera diferenças raciais e étnicas. Tem sido notado que a mensuração da distribuição de gordura é mais preditiva de saúde, conforme LEAN et al. (1998) e RANKINEN et al. (1999).

Deve-se salientar, a princípio, que não existe avaliação perfeita para sobrepeso e obesidade. Há que se distinguir o diagnóstico da desnutrição e da obesidade. No caso da desnutrição, o emprego da relação peso/altura, expressa por meio do IMC, encontra sustentação no fato de que ambos os compartimentos da massa corporal - o tecido adiposo e a massa magra - são afetados pela desnutrição. Ademais, a relação entre baixos valores de IMC e maior ocorrência de morbi-mortalidade e alterações da função reprodutiva tem sido demonstrada em estudos empíricos (GARCIA\& KENNEDY, 1994; MONDINI \& MONTEIRO, 1998).

O fato de que valores elevados de IMC, em tese, não façam distinção entre acúmulo de tecido adiposo (obesidade) e aumentos na massa magra torna menos seguro o emprego desse índice no diagnóstico da obesidade. Ainda assim, recomenda-se o seu uso diante das dificuldades operacionais relacionadas ao emprego de medidas diretas da composição corporal (MONDINI \& MONTEIRO, 1998).

Apesar de os estudos existentes a respeito terem apontado problemas ocasionados pelo excesso de peso no homem, há um evidente descaso com os animais de companhia. Há uma total ausência de métodos precisos e objetivos para determinar qual é o peso ideal e se o animal está acima ou abaixo do peso.

O propósito maior de saber identificar o grau da obesidade do cão é evitar o comprometimento da função fisiológica normal e os problemas metabólicos, cirúrgicos e/ou mecânicos acarretados pelo excesso de peso (CARAM, 1999).

Este trabalho propõe a adaptação, para cães, do índice de massa corporal utilizado em humanos, buscando-se obter um critério simples e objetivo de quantificação de massa corporal para esta espécie. Foi utilizada a nomenclatura estatura para referir-se ao comprimento da coluna e dos membros posteriores. Por fim, realizou-se uma correlação com o peso e assim foram obtidas informações numéricas de cada faixa de condição corporal do cão.

\section{MATERIAL E MÉTODOS}

Foram utilizados 246 cães domésticos, machos ou fêmeas, adultos, de diversos pesos e raças ou mesmo mestiços, recebidos para consulta no Hospital Veterinário Universitário da Universidade 
Tabela 1 - Graduação de condição corporal para caninos, proposta por LAFLAMME (1997).

\begin{tabular}{|c|c|c|}
\hline Condição & Grau & Características \\
\hline \multirow{12}{*}{ Subalimentado } & \multirow{4}{*}{1} & - Costelas, vértebras lombares, ossos pélvicos e saliências ósseas visíveis à distância \\
\hline & & - Não há gordura corporal \\
\hline & & - Perda evidente de massa muscular \\
\hline & & - Costelas, vértebras e ossos pélvicos facilmente visíveis \\
\hline & \multirow{4}{*}{2} & - Não há gordura palpável \\
\hline & & - Algumas saliências podem estar visíveis \\
\hline & & - Perda mínima de massa muscular \\
\hline & & - Costelas facilmente palpáveis podem estar visíveis sem gordura palpável \\
\hline & \multirow{4}{*}{3} & - Visível o topo das vértebras lombares \\
\hline & & - Ossos pélvicos começam a ficar visíveis \\
\hline & & - Cintura e reentrâncias abdominais evidentes \\
\hline & & - Costelas facilmente palpáveis com mínima cobertura de gordura \\
\hline \multirow[t]{5}{*}{ Ideal } & \multirow[t]{2}{*}{4} & - Vista de cima, a cintura é facilmente observada \\
\hline & & - Reentrância abdominal evidente \\
\hline & \multirow{3}{*}{5} & - Costelas palpáveis sem excessiva cobertura de gordura \\
\hline & & - Abdômen retraído quando visto de lado \\
\hline & & - Costelas palpáveis com leve excesso de cobertura \\
\hline \multirow[t]{14}{*}{ Sobrealimentado } & \multirow[t]{4}{*}{6} & - Cintura é visível quando vista de cima, mas não é acentuada \\
\hline & & - Reentrância abdominal aparente \\
\hline & & - Costelas palpáveis com dificuldade \\
\hline & & - Pesada cobertura de gordura \\
\hline & \multirow[t]{4}{*}{7} & - Depósito de gordura evidente sobre a área lombar e base da cauda \\
\hline & & - Ausência de cintura ou apenas visível \\
\hline & & - Reentrância abdominal pode estar presente \\
\hline & & $\begin{array}{l}\text { - Impossível palpar as costelas situadas sob cobertura muito densa ou palpável somente } \\
\text { com pressão acentuada }\end{array}$ \\
\hline & \multirow[t]{4}{*}{8} & - Pesado depósito de gordura sobre área lombar e base da cauda \\
\hline & & - Cintura inexistente \\
\hline & & - Não há reentrância abdominal, podendo existir distensão abdominal evidente \\
\hline & & - Maciços depósitos de gordura sobre o tórax, espinha e base da cauda \\
\hline & \multirow[t]{2}{*}{9} & - Depósitos de gordura no pescoço e membros \\
\hline & & - Distensão abdominal evidente \\
\hline
\end{tabular}

Federal de Santa (UFSM) - RS e coletados durante a campanha de vacinação anti-rábica, realizada nas cidades de São Sepé/RS e São Pedro do Sul/RS, durante o ano de 2006.

Todos os animais tiveram registrados, na respectiva ficha clínica, o peso, o comprimento da coluna adicionado ao comprimento dos membros pélvicos e a raça (ou tipo físico daqueles sem raça definida - SRD). Nessa ficha, adicionou-se um campo, referente à condição corporal do cão, proposta por LAFLAMME (1997).

Para a mensuração da estatura do cão (referente à altura utilizada na fórmula do IMC humano), utilizou-se uma fita métrica flexível. Considerou-se como pontos de referência a extensão entre a base da nuca (articulação atlanto-occipital) e o solo imediatamente atrás dos membros posteriores, passando e apoiando a fita sobre a base da cauda (última vértebra sacral), ficando a fita exatamente medial às tuberosidades ilíacas, sobre o dorso do animal, conforme figura 1.

Para a aferição do peso, foi utilizada uma balança digital com capacidade de até 100 quilos. Depois de obtidos os dados, fez-se uma equação aritmética a partir do índice de massa corporal humano:

Índice de Massa Corporal Canino (IMCC) = peso corporal $(\mathrm{kg})$ (estatura em m) $)^{2}$

A condição corporal proposta por LAFLAMME (1997) foi avaliada por dois profissionais da área que utilizaram sempre os mesmos critérios. Cabe ressaltar que a avaliação clínica foi realizada por dois avaliadores para que fosse diluída qualquer tendência na obtenção dos dados subjetivos, esclarecendo que estes desconheciam qualquer dado sobre peso, estatura ou qualquer outra proposta de avaliação. Dessa forma, foi possível realizar um cruzamento de dados, 


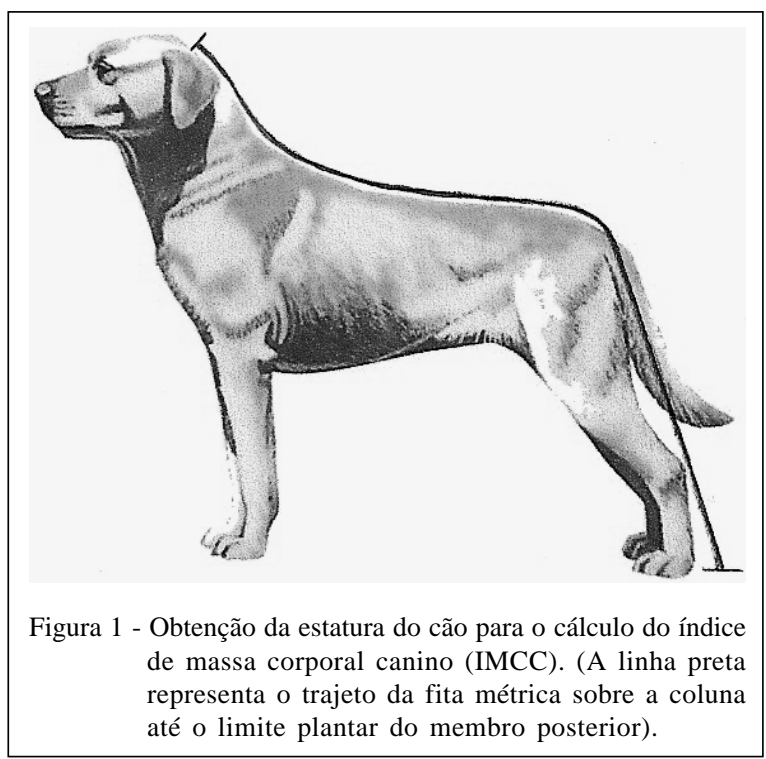

correlacionando o IMCC obtido e a avaliação subjetiva.

\section{RESULTADOS E DISCUSSÕES}

Considerando o cruzamento de dados entre a equação do IMCC e a condição corporal do animal, avaliada clinicamente, chegou-se a um quadro dos 246 animais. No IMC de humanos existem apenas quatro grupos principais que informam a condição física, sendo eles: abaixo do peso, peso ideal, acima do peso e obeso. A partir deste raciocínio, optou-se por também agrupar as avaliações dos cães nas mesmas quatro condições, conforme demonstrado na tabela 2.

Todos os cães foram classificados dentro de uma das nove avaliações sugeridas por LAFLAMME (1997). Aqueles pertencentes ao grupo 1, 2 ou 3 foram agrupados na condição 1, que se refere aos cães abaixo do peso ideal. Da mesma forma, cães avaliados como pertencentes aos grupos 4 e 5 formaram a condição 2 (dentro do peso ideal); 6 e 7 compuseram a condição 3 (acima do peso ideal) e 8 e 9 a condição 4 (obesos) (Tabela 2).

Através do programa estatístico SAS SYSTEM (BARR et al., 1979), os dados foram analisados e se chegou ao IMCC referente a cada uma das quatro condições (Tabela 2). Correlacionando a coluna “condição" com a coluna "IMCC”, observouse que o grau de correlação foi altamente significativo.

Observando a população estudada (246 cães), a grande maioria (53,4\%) dos cães estavam dentro da faixa da normalidade de peso, sendo que 7,1\% encontravam-se abaixo do peso, 33,6 \% acima do peso e apenas 5,9 \% dos cães avaliados estavam obesos. Estima-se que estes percentuais representem o universo de cães sadios da região.

O grande benefício do IMCC na medicina veterinária é saber quantos quilos de fato o animal deve perder ou ganhar. Diante disso, torna-se mais palpável para o proprietário a meta da dieta. Já para o veterinário, o acompanhamento do animal passa a ter um dado matemático e não sujeito às interpretações subjetivas.

Contudo, o uso do IMC deixa de levar em conta a composição proporcional do corpo ou a distribuição de gordura corporal. Mais especificamente, outros fatores, além do excesso de gordura corporal osso, massa muscular e até mesmo o aumento de volume plasmático induzido pelo treinamento com exercícios - afetam os números da equação para o IMC. Um IMC alto poderia dar origem a uma interpretação incorreta de gordura excessiva em indivíduos magros, porém com musculatura excessiva, em virtude da constituição genética ou do treinamento com exercícios. Um exemplo disso são os atletas fisiculturistas, que

Tabela 2 - Quadro referente aos dados obtidos neste trabalho. Na primeira coluna, têm-se as quatro condições utilizadas no IMC humano. Na segunda coluna, as nove escalas de avaliações dos escores corporais de cães, proposta por Laflamme (1997). Na terceira coluna, encontram-se os dados de IMCC obtidos neste experimento, correspondente às nove condições de Laflamme (1997) e, na última coluna, têm-se os IMCC médios obtidos neste trabalho, agrupados nas quatro condições referentes ao IMC humano.

\begin{tabular}{lccc}
\hline Condição & Avaliação (Laflamme) & IMCC da avaliação* & IMCC médio da condição* e intervalo \\
\hline \multirow{3}{*}{1 - Abaixo do peso } & 1 & 06,470 & 10,527 \\
& 2 & 08,115 & $($ abaixo de 11,7) \\
2 - Peso ideal & 3 & 11,693 & 13,497 \\
& 4 & 11,867 & $($ entre 11,8 e 15) \\
3 - Acima do peso & 5 & 14,304 & 16,378 \\
& 6 & 15,951 & $($ entre 15,1 e 18,6) \\
4 - Obeso & 7 & 17,594 & 20,177 \\
& 8 & 19,695 & (acima de 18,7) \\
\hline
\end{tabular}

* Resultados obtidos pelo programa SAS SYSTEM (BARR et al., 1979). 
possuem um IMC elevado, caracterizando obesidade, porém apresentam porcentagens de gordura corporal abaixo dos níveis normais (MCARDLE et al., 2003).

Da mesma forma, em cães, observou-se grande dificuldade em agrupar animais de raças tão distintas e com área corporal tão variada. Entretanto, os resultados obtidos foram considerados extremamente válidos por serem dados médios objetivos de uma população de cães, já que o diagnóstico de obesidade na clínica de pequenos animais, especificamente no cão, é extremamente subjetivo.

De acordo com o porte, pode-se observar, nos cães, as diferenças na necessidade energética, no peso ao nascimento e no tamanho da ninhada, na velocidade e na duração do crescimento, no tamanho relativo do trato digestivo, na expectativa de vida, na predisposição a algumas doenças (distocia, obesidade, dilatação gástrica e volvo) e no estilo de vida. Estas diferenças tornam possível dividir os cães em raças pequenas (1-10kg), médias (10-25kg) e grandes (> 25kg) e explicar as variações das necessidades dietéticas entre as raças (BIOURGE \& PIBOT, 2005).

O tabela 2 apresenta dados de IMCC referentes exclusivamente àqueles cães cujas raças pertencem ao porte médio, ou seja, tipos físicos cuja média de peso varia entre 10 e $25 \mathrm{~kg}$. Entretanto, pôdese observar neste estudo, com boa margem de segurança, que o IMCC para as raças de grande porte apresenta um acréscimo de $20 \%$ do IMCC daqueles de médio porte. Percebeu-se ainda que para os tipos físicos miniaturas (até $10 \mathrm{~kg}$ ) ocorre uma diminuição em 10\% no IMCC daqueles de médio porte.

Para exemplificar a situação citada acima, basta imaginar um cão da raça boxer (grande porte), medindo $1,10 \mathrm{~m}$ de estatura (coluna + altura dos posteriores) e pesando 20,8kg. Aplicando a fórmula IMCC=peso estatura-2, obtem-se um valor de IMCC=17,25. Deste valor, basta diminuir 20\% e comparar com a tabela 2 para observar em qual condição o animal se encontra. Neste exemplo, ao se diminuir $20 \%$ no IMCC, obtem-se o valor de 13,80, o que significa que o animal está no peso ideal. Sendo o animal um cão de pequeno porte, bastaria aumentar 10\% (ao invés de diminuir 20\%) para chegar aos valores da tabela 2 .

Eventualmente poderá existir pacientes com IMCC relativamente fora dos dados encontrados neste trabalho. Porém, caberá ao clínico interpretar os resultados, considerando fatores do animal que possam diferir do padrão normal para a população. Como exemplo, as raças como a Pit Bull, a qual apresenta um grau de musculatura avantajado quando comparado com outras raças. Isso pode resultar em IMCC compatível com obesidade (situação idêntica àquelas pessoas fisiculturistas). Fazendo um paralelo, o IMC de humanos foi desenvolvido para indivíduos americanos, o que poderá apresentar diferenças consideráveis quando aplicado em asiáticos (MCARDLE et al., 2003).

Dessa forma, o IMCC poderá servir também como parâmetro ao desenvolvimento de atividades fisícas controladas para cães, sendo estas com fins de condicionamento físico a animais atletas ou aqueles que necessitem do exercício como terapia. Para tanto, a tabela 2 apresenta o resultado final deste trabalho com as faixas aceitáveis de IMCC em cada condição corporal e permite ao médico veterinário chamar a atenção do proprietário do animal para o estado nutricional do mesmo e a necessidade de acompanhamento

\section{CONCLUSÃO}

Diante dos dados obtidos, é permitido concluir que a medida da coluna vertebral adicionada ao comprimento do membro pélvico é um parâmetro viável em cães para substituir a altura, utilizada em humanos. Conclui-se também que o IMCC ideal para cães cujo tipo físico é de médio porte (peso médio entre 10 e $25 \mathrm{~kg}$ ) compreende valores entre 11,8 e $15 \mathrm{~kg} \mathrm{~m}^{-2}$. Um animal que possua o peso $20 \%$ acima ou abaixo do peso ideal pode ser considerado fora do peso.

Sugere-se que o IMCC possa ser utilizado como um dado médio de cálculo para tratamentos e para orientar treinamentos físicos de caninos.

\section{REFERÊNCIAS}

ANJOS, L.A. Índice de massa corporal (massa corporal, estatura-2) como indicador do estado nutricional de adultos. Revista Saúde Pública, v.26, n.6, p.431-436, 1992.

BARR, ANTHONY J et al. SAS Programmer's guide. Carry, 1979.

BIOURGE, V.; PIBOT, P. A nutrição sob medida. Paris: Royal Canin, 2005. 12p

BURKHOLDER, W.J.; TOLL, P.W. Obesity. In: HAND, M.S. et al. Small animal clinical nutrition. 4.ed. Topeka: Mark Morris Institute, 2000. p.401-430.

CARAM, A. L. G. “Obesidade” cães e gatos. São Paulo: Palo Verde, 1999. Capturado em 10 mai. 2006. Online. Disponível na Internet http://www.paloverde.com.br/dicas/dicas30.htm.

GARCIA, M.; KENNEDY, E. Assessing linkages between body mass index and morbidity in adults: evidences from four developing countries. European Journal of Clinical Nutrition, v.48, Suppl.3, p.90-97, 1994. 
LAFLAMME, D.P. Development and validation of a body condition score system for dogs. Canine Practice, v.22, p.10-15, 1997.

LEAN, M.E. et al. Impairment of health and quality of life in people with large waist circumference. Lancet, v.351, p.853856, 1998.

MENTZEL, R.E. et al. Obesidade no cão e no gato: abordagem comportamental. Paris: Royal Canin, 2006. 55p.

MCARDLE, W.D. et al. Fisiologia do exercício: energia, nutrição e desenvolvimento humano. 5.ed. Rio de Janeiro: Guanabara Koogan, 2003. 1113p.

MONDINI, L.; MONTEIRO, C.A. Relevância epidemiológica da desnutrição e da obesidade em distintas classes sociais: métodos de estudo e aplicação à população brasileira. Revista Brasileira de Epidemiologia, v.1 n.1, p.28-39, 1998.

MONTEIRO, P.O.A. et al. Diagnóstico de sobrepeso em adolescentes: estudo do desempenho de diferentes critérios para o Índice de Massa Corporal. Revista de Saúde Pública, v.34, n.5 p.506-513, 2000.

MONTILLA, R.N.G. et al. Avaliação do estado nutricional e do consumo alimentar de mulheres no climatério. Revista de Associação Medica Brasileira, v.49, n.1, p.91-95, 2003.

NELSON, R.; COUTO, C.G. Medicina interna de pequenos animais. 2.ed. Rio de Janeiro: Guanabara Koogan, 2001. 1324p.

RANKINEN, T. et al. The prediction of abdominal visceral fat level from body composition and anthropometry: ROC analysis. International Journal of Obesity and Related Metabolic Disorders, v.23, n.8, p.801-809, 1999.

SALIOLA, M. Gordinhos não saudáveis. mar. 2001. Capturado em 12 mai. 2006. Online. Disponível na Internet http://www.dogtimes.com.br/obesidade.htm.

STELLA, R. Índice de massa corporal (IMC), mar. 2002 Capturado em 01 dez. 2006. Online. Disponível na Internet ht tp: / / www 1.uol.com.br/cyberdiet/colunas/ 020309_nut_x_conhecer_imc.htm 